\title{
Norepinephrine effects on the encoding and consolidation of emotional memory: improving synergy between animal and human studies
}

Running title: Norepinephrine effects on encoding and consolidation

Benno Roozendaal \& Erno J. Hermans

Department of Cognitive Neuroscience, Radboud university medical center, 6500 HB Nijmegen, The Netherlands

Donders Institute for Brain, Cognition and Behaviour, Radboud University, 6525 EN Nijmegen, The Netherlands

Corresponding author:

Benno Roozendaal

Department of Cognitive Neuroscience, Radboud university medical center

P.O. Box 9101, 6500 HB Nijmegen, The Netherlands

e-mail: Benno.Roozendaal@radboudumc.nl 


\begin{abstract}
Noradrenergic activity is important for emotional enhancement of memory. Although findings from both animal and human research provide extensive support for this general conclusion, there are some important, but often ignored, differences between these research lines. Whereas animal experiments mostly employ pharmacological manipulations in the post-learning phase to investigate the effects of sustained noradrenergic activation on the consolidation of long-term memory, neuroimaging studies in humans typically focus on much more dynamic changes in noradrenergic activity during the actual encoding of information. In this paper we discuss the possibility that these two types of noradrenergic effects interact in enhancing memory for emotionally arousing experiences, and explain how elucidating this mechanism might improve synergy between animal and human research.
\end{abstract}




\section{Introduction}

Extensive evidence indicates that noradrenergic activity is enhanced by emotionally arousing training conditions [1]. The hypothesis that norepinephrine plays a role in learning and memory emerged more than four decades ago when Kety [2] suggested that adrenergic catecholamines, such as epinephrine or norepinephrine, released by certain emotional states may serve "to reinforce and consolidate new and significant sensory patterns in the neocortex." The initial experiments investigating this general hypothesis examined the effects of systemic administration of adrenergic drugs on learning and memory [3]. The findings of Gold and van Buskirk [4] were among the first to suggest the involvement of central norepinephrine in memory. Many subsequent studies in rodents provided extensive support for the hypothesis that norepinephrine or a $\beta$-adrenoceptor agonist infused into the basolateral amygdala (BLA) or other brain regions such as the hippocampus or prefrontal cortex enhances long-term memory of emotionally arousing training experiences [1,5,6]. Human research generally supports the conclusions of animal studies indicating that an activation of the noradrenergic system is associated with better memory and that this influence involves the amygdala $[7,8]$.

There are, however, some important but frequently overlooked methodological differences between the animal and human studies. Whereas most animal research has used post-learning pharmacological manipulations to induce sustained changes in noradrenergic activity during the consolidation phase of memory, human neuroimaging studies typically focus on much more dynamic, i.e., phasic, changes in noradrenergic activity during the actual encoding of emotional experiences into memory. While phasic noradrenergic signaling is centrally mediated through rapid increases in firing rates of locus coeruleus neurons [9], sustained noradrenergic activity after 
encoding, at least partially, involves activation of peripheral adrenal stress hormone release: Both epinephrine [10] and glucocorticoids [11] trigger a tonic increase in noradrenergic activity in the amygdala. Such peripheral stress hormone effects on sustained noradrenergic activity might not only be produced as a result of tonic activation of noradrenergic neurons in the locus coeruleus [12] but also involve the activation of noradrenergic cell groups in the nucleus of the solitary tract [10] or an indirect stimulation of norepinephrine levels by inhibiting norepinephrine-reuptake mechanisms [13]. In this paper, we will argue that these phasic and sustained increases in noradrenergic activity might interact in enhancing memory for emotionally arousing experiences. The specific and phasic noradrenergic activation during memory encoding might make these memory traces amenable to the memory-enhancing effects of a more sustained noradrenergic activation in the post-learning consolidation phase. Notably, this sustained post-learning noradrenergic activity may also have no, or even opposite, effects when not preceded by phasic noradrenergic signaling during encoding.

\section{Norepinephrine actions on memory consolidation}

Animal studies have provided extensive evidence that noradrenergic activation, arising from catecholaminergic cell bodies in the locus coeruleus and the nucleus of the solitary tract, is critically involved in memory consolidation [14]. For example, norepinephrine or $\beta$-adrenoceptor agonists administered into the BLA immediately after an emotionally arousing training experience induce dose-dependent enhancement of memory consolidation [15-21]. Similar norepinephrine infusions into the hippocampus, entorhinal cortex or posterior parietal cortex enhance memory consolidation for inhibitory avoidance training when given even up to 6 hours after training 
$[22,23]$. Conversely, post-training infusions of $\beta$-adrenoceptor antagonists impair retention and block the memory-enhancing effects of co-administered norepinephrine $[19,20]$. In addition to $\beta$ adrenoceptor influences, $\alpha_{1}$-adrenoceptor agonist infusions into the BLA after training also enhance memory consolidation [24]. The $\alpha_{1}$-adrenoceptor-induced memory enhancement most likely involves an interaction with $\beta$-adrenoceptors, as post-training intra-BLA infusions of a $\beta$ adrenoceptor antagonist block the memory enhancement produced by activation of $\alpha_{1^{-}}$ adrenoceptors [25]. Undoubtedly, there are many other influences on memory consolidation provided by adrenomedullary and adrenocortical stress hormones, other neurotransmitter systems as well as local circuit neurons. Although memory modulation can be achieved by manipulation of any of these components, there are many interactions between the noradrenergic system and these neuromodulatory influences [14]. For example, drugs and hormones that enhance memory consolidation potentiate training-induced increases in norepinephrine levels in the amygdala whereas drugs that impair consolidation decrease amygdala norepinephrine levels $[10,11]$. Moreover, an attenuation of noradrenergic activity with a $\beta$-adrenoceptor antagonist administered into the BLA blocks the memory-modulatory influences of post-learning stress hormone or neurotransmitter administration [26,27].

Most pharmacological studies have used targeted administrations of norepinephrine or noradrenergic agents into relevant brain regions [14]. As these infusions are given mostly after the training experience, the norepinephrine likely induces a prolonged increase in noradrenergic activity during the consolidation phase of memory processing. In support of this view, in vivo microdialysis studies showed that noradrenergic activity in the amygdala is elevated for approximately 2 hours after an aversive learning experience [28] (Figure 1). Further and 
importantly, in rats given inhibitory avoidance training, the magnitude of training-induced increases in amygdala norepinephrine levels assessed after the training correlates very highly with the rats' subsequent long-term retention performance, providing additional evidence that relatively long-lasting elevations in noradrenergic activity after the actual learning experience might be required to induce enhancement of memory consolidation. Such noradrenergic enhancement of amygdala activity modulates the consolidation of memory for many kinds of emotionally arousing training experiences [29]. Consistent with this evidence, post-training noradrenergic manipulation of BLA activity can influence neuroplasticity and information storage processes in other brain regions known to be involved in memory processing, including the hippocampus, caudate nucleus and insular cortex $[1,14,30]$.

Human research demonstrated that systemic administration of the noradrenergic stimulant yohimbine before learning enhances memory [8]. Conversely, blocking the effects of endogenous norepinephrine using pre-learning administration of the $\beta$-adrenoceptor antagonist propranolol results in impaired memory for emotionally arousing events [7]. Such noradrenergic effects are centrally mediated, as the $\beta$-adrenoceptor blocker nadolol, which cannot cross the blood-brain barrier, does not have the same effect as propranolol [31]. Studies involving noradrenergic manipulations before encoding, however, cannot dissociate effects on encoding from effects on consolidation. Findings converge, however, with studies using post-learning exposure to psychosocial [32,33], physiological [34-37], and negatively emotionally arousing [38] stressors, which are also associated (albeit not uniquely) with sustained noradrenergic activity. Most direct evidence for noradrenergic involvement in memory consolidation comes from studies showing similar memory-enhancing effects following post-learning administration of yohimbine [39] and 
epinephrine [40].

\section{Norepinephrine actions on memory encoding}

While pharmacological work in rodents mainly focused on the role of norepinephrine in enhancing the consolidation of memories, it is evident that norepinephrine must also play an important role during memory encoding. A large body of research implicates noradrenergic activation by the locus coeruleus in regulating attention, and thus in regulating sensory intake that is to be encoded. Work in monkeys has shown that locus coeruleus neurons exhibit two distinct modes of activity [9]. Tonic discharge rates of locus coeruleus neurons are positively associated with levels of arousal, and peak in states of stress [12]. By contrast, phasic locus coeruleus activity is related to levels of arousal in an inverted U-shaped fashion, with most efficient signaling at moderate levels of arousal [9]. These brief neuromodulatory signals are thought to send "interrupt" signals to currently active functional networks [41], resulting in disengagement from current attentional sets [9], rapid rearrangement of functional networks [42], and a reorienting of attention toward salient environmental information [43]. In humans, phasic norepinephrine has been linked to electrophysiological correlates of sensory processing [44], and to allocation of attention to both emotional and neutral stimuli [45]. Noradrenergic activity has furthermore been associated with activation of the "salience" network [46]. This network, which was delineated using measures of intrinsic functional connectivity in humans, integrates various neurocognitive functions relevant to processing salient stimuli, including attentional reorienting, homeostatic regulation, and interoception [47]. It is anchored in dorsal anterior cingulate cortex and anterior insula, but also involves the amygdala $[47,48]$. 
Human research using functional neuroimaging, which is particularly suited to study phasic activity in response to stimuli, has therefore focused on the amygdala during processing and encoding of emotional stimuli in humans. Amygdala activity during encoding, measured using PET, predicts memory for emotionally arousing experiences $[49,50]$. With BOLD-fMRI, such associations between amygdala activity and subsequent memory were extended to phasic, trialby-trial, variation in success of emotional memory formation [51-53]. Connectivity between amygdala and hippocampus increases during encoding [54] and predicts subsequent memory [55]. Enhanced mnemonic activity in hippocampus for emotional stimuli furthermore depends on the integrity of the amygdala [56].

While neuroimaging studies into the amygdala cannot establish noradrenergic involvement, pharmacological studies have provided more direct evidence for the key role of norepinephrine in facilitating the amygdala's role during memory formation. For instance, ß-adrenoceptor blockade using propranolol during encoding reduces both the emotional memory enhancement effect [7] and the amygdala response to emotional material $[57,58]$, although it may also reduce neutral memory [59]. Enhancing noradrenergic activity using the selective norepinephrinereuptake inhibitor reboxetine has the opposite effect of increasing the amygdala responsiveness [60]. The fact that these effects are specific to relevant brain regions makes it unlikely that they are caused by cardiovascular artefacts. Finally, carriers of a common functional deletion in the gene coding for the presynaptic $\alpha_{2 b}$-adrenoceptor (ADRA2B), who have elevated norepinephrine levels, exhibit stronger amygdala responses to aversive stimuli [61], stronger increases in phasic amygdala responses during stress [62], and stronger enhancement of emotional memory [63]. Thus, there is abundant evidence demonstrating that (correlates of) phasic noradrenergic activity 
and amygdala activity predict enhanced memory formation for emotional material in humans.

\section{Interactions between noradrenergic actions during encoding and consolidation}

Up until recently, encoding and consolidation processes have been studied in relative isolation, largely along the lines of human versus animal research, respectively. The reason for this is that research in animals has often relied on targeted pharmacological manipulations in the postlearning consolidation phase which are too invasive to employ in humans. Contrariwise, the noninvasive techniques available in humans are more suited to study mnemonic processes during encoding, because most conventional methods for human functional neuroimaging are developed for detecting time-locked, evoked neuronal activity. Human functional neuroimaging cannot be easily applied to latent, temporally unpredictable neural processes such as those that may occur during consolidation. Therefore, research into encoding and consolidation processes has not yet been optimally integrated, leaving important open questions regarding the relationship between these two processes. Are they simply additive, with more activity in either leading to better memory, or could it be that they interact: Can activity at encoding determine the fate of a memory during the consolidation phase? Before turning to this question, we will first describe recent interdisciplinary attempts at connecting encoding and consolidation processes in animal and human work.

The first attempts at connecting encoding and consolidation processes have been made in rodent electrophysiology. These studies have shown increased synchronization of neuronal oscillations between amygdala and hippocampus, for instance, during acquisition and expression of conditioned fear [64], but also after immobilization stress [65], both of which are experiences 
that likely trigger sympathetic and noradrenergic arousal [66]. Importantly, amygdalahippocampal, but also amygdala-medial prefrontal, theta synchronization is increased during sleep after similarly arousing experiences, and this increase predicts memory one day later [67]. Although no studies to date have explicitly linked these effects to noradrenergic activity, arousing experiences due to reward or novelty furthermore strengthen spontaneous reactivation ("replay") of waking patterns of hippocampal activity during post-learning awake rest periods $[68,69]$. Note that both "offline" rest periods after learning and sleep are seen as critical periods for memory consolidation. In conclusion, it appears that neural circuits underlying encoding and consolidation at least partly overlap.

Following this logic, recent work using functional neuroimaging in humans has attempted to capture the same phenomena in humans by investigating post-learning reactivations of encoding patterns of neuronal activity within, and connectivity between, the regions implicated in rodent work. Early studies demonstrated that hippocampal-neocortical connectivity indeed persists during rest following encoding of neutral memories [70,71]. Persistence of this connectivity [70] as well as persistence of hippocampal [72] and entorhinal [73] activity patterns during postencoding rest was furthermore linked to subsequent memory recall. For emotional memories, it was shown that enhanced amygdala-hippocampal connectivity persists during a waking period (in the range of approximately 0-30 minutes) after fear learning, and predicts long-term expression of fear [74]. Furthermore, fear learning enhances hippocampally driven spontaneous reactivation of patterns of activity in neocortical representational regions within the ventral visual stream, showing that recently acquired memory representations reactivate spontaneously during post-encoding rest when associated with emotionally arousing events through fear conditioning 
[75]. Note that, although a direct link with norepinephrine remains to be shown, these reactivations occur within a time frame that falls within the period during which noradrenergic activity remains elevated following arousing footshocks in rodents (see Figure 1).

A critical open question is what the nature is of the relationship between phasic noradrenergic activity during encoding and sustained activity during consolidation. Several studies have provided converging evidence for the notion that the memory-modulatory effects of sustained post-learning noradrenergic activity depend on phasic noradrenergic activity during encoding. Animal studies typically employ emotionally arousing training conditions and consistently find memory-enhancing effects of post-learning noradrenergic activation (see section on memory consolidation above), but human studies that have applied experimental stress induction procedures after memory encoding have shown a selective enhancement of consolidation for information that was arousing during encoding [32,34-37] (but see [33]). A recent study showed that stronger cortisol reactivity to post-learning stress induction was associated with stronger subsequent memory effects in amygdala and hippocampus [76], which suggests that postlearning elevation of stress hormones makes memories more dependent on phasic activity in medial temporal lobe structures during encoding. Beneficial effects of post-learning manipulations have also been reported with systemic administration of epinephrine [40] and hydrocortisone $[77,78]$. It appears that such post-learning manipulations are only effective within a limited time window [38], which roughly corresponds with the time window in which "replay"like effects were found $[74,75]$. Notably, beneficial effects of post-learning manipulations on retention of emotionally arousing material reported in the human literature are often accompanied by a numerical decrease for neutral material studied within the same context 
$[34,35,40,78]$. Such a selective mnemonic impairment for neutral material is particularly evident in the E-1 paradigm, in which memory for neutral items is impaired when directly followed by an emotionally arousing oddball [79], but only when non-salient (i.e., task-irrelevant) [80]. One explanation for such dual post-learning effects of norepinephrine was recently proposed by Mather et al. [81], who argue that such effects are due to bidirectional local interactions of norepinephrine with glutamatergic activity in brain regions involved in representing the items.

Converging evidence from neuropsychological and rodent studies, however, point toward another explanation, namely that the amygdala continues to play a critical role in both the enhancement of consolidation of emotionally arousing information as well as the impairment of the consolidation of neutral information after post-learning arousal. For instance, patients with Urbach-Wiethe disease (UWD), which causes progressive calcification particularly of the BLA [82], not only fail to show a normal emotional enhancement effect [83], but also lack the selective impairment for neutral stimuli preceding emotionally arousing oddballs in E-1 paradigms [79]. This effect cannot simply be explained by general impairment of noradrenergic signaling, as UWD patients appear to exhibit normal arousal responses to, for instance, unconditioned stimuli $[84,85]$. Furthermore, rodent work shows that infusion of the $\beta$-adrenoceptor agonist clenbuterol into the BLA triggers a long-term increase in excitability of hippocampal neurons, but only when administered after an emotionally arousing inhibitory avoidance experience. Critically, clenbuterol infusion has an opposite effect in home-cage control animals which did not undergo the arousing training experience [30]. Noradrenergic activity in the amygdala during memory encoding also appears to determine the direction of the effect of post-learning administration of stress hormones on memory. For instance, the synthetic glucocorticoid dexamethasone, which 
increases norepinephrine levels in brain [11], administered post-training enhances long-term memory for inhibitory avoidance training in rats with intact noradrenergic signaling in the BLA, but has an opposite effect when noradrenergic signaling in the BLA during training is blocked using a $\beta$-adrenoceptor antagonist [27]. Figure 2 summarizes the model of how the effect of postlearning stress exposure and associated sustained increases in noradrenergic activity on memory consolidation might interact with phasic noradrenergic activity in the amygdala during memory encoding.

\section{Conclusions and future directions}

In conclusion, a critical analysis of the extant literature on the effects of norepinephrine on memory shows a divide between animal research, which has mainly investigated the consolidation phase, and human research, which has traditionally focused on encoding processes. As we outlined, a synergy of these two research lines is beginning to emerge. This is important particularly because noradrenergic effects on encoding and consolidation are not additive but interactive: Noradrenergic signaling during encoding determines the direction of noradrenergic effects during consolidation. Furthermore, we have argued that the amygdala, as a "nexus" within distinct large-scale neural networks [86], plays a critical role in both phases. Future research should leverage the strengths of both human and animal research: The ability of human functional neuroimaging to map interactions within large-scale neural systems and the ability to employ invasive novel technologies such as optogenetics in rodents. A synergy of these two fields will help us in understanding the neural mechanisms underlying the mnemonic consequences of emotional experiences. 


\section{Acknowledgments}

BR is supported by a Topfund grant from Radboud University and EJH is supported by a grant from the European Research Council (ERC-2015-CoG 682591).

\section{References}

1. McGaugh JL: The amygdala modulates the consolidation of memories of emotionally arousing experiences. Annu. Rev. Neurosci. 2004, 27:1-28.

2. Kety S: Brain catecholamines, affective states and memory. In The Chemistry of Mood, Motivation, and Memory. Plenum Press; 1972:65-80.

3. Archer T: DSP4 (N-2-chloroethyl-N-ethyl-2-bromobenzylamine), a new noradrenaline neurotoxin, and stimulus conditions affecting acquisition of two-way active avoidance. J Comp Physiol Psychol 1982, 96:476-490.

4. Gold PE, van Buskirk R: Effects of alpha- and beta-adrenergic receptor antagonists on post-trial epinephrine modulation of memory: relationship to post-training brain norepinephrine concentrations. Behav Biol 1978, 24:168-184.

5. McGaugh JL: Memory--a century of consolidation. Science 2000, 287:248-251.

6. McGaugh JL, Roozendaal B: Role of adrenal stress hormones in forming lasting memories in the brain. Curr. Opin. Neurobiol. 2002, 12:205-210.

**7. Cahill L, Prins B, Weber M, McGaugh JL: Beta-adrenergic activation and memory for emotional events. Nature 1994, 371:702-704.

A seminal study providing first evidence that blockade of noradrenergic activity in humans selectively impairs long-term memory of emotional material in humans.

8. O'Carroll RE, Drysdale E, Cahill L, Shajahan P, Ebmeier KP: Stimulation of the noradrenergic system enhances and blockade reduces memory for emotional material in man. Psychol. Med. 1999, 29:1083-1088.

*9. Aston-Jones G, Cohen JD: An integrative theory of locus coeruleus-norepinephrine function: adaptive gain and optimal performance. Annu. Rev. Neurosci. 2004, 28:403450.

10. Williams CL, Men D, Clayton EC, Gold PE: Norepinephrine release in the amygdala after 
systemic injection of epinephrine or escapable footshock: contribution of the nucleus of the solitary tract. Behav. Neurosci. 1998, 112:1414-1422.

11. McReynolds JR, Donowho K, Abdi A, McGaugh JL, Roozendaal B, McIntyre CK: Memoryenhancing corticosterone treatment increases amygdala norepinephrine and Arc protein expression in hippocampal synaptic fractions. Neurobiol. Learn. Mem. 2010, 93:312-321.

12. Valentino RJ, van Bockstaele E: Convergent regulation of locus coeruleus activity as an adaptive response to stress. Eur. J. Pharmacol. 2008, 583:194-203.

13. Gründemann D, Schechinger B, Rappold GA, Schömig E: Molecular identification of the corticosterone-sensitive extraneuronal catecholamine transporter. Nat. Neurosci. 1998, $1: 349-351$.

**14. Roozendaal B, McGaugh JL: Memory modulation. Behav. Neurosci. 2011, 125:797-824.

An extensive review of the animal and human literature on the role of the amygdala and its influence on other brain regions in regulating memory consolidation of emotional experiences.

15. Liang KC, McGaugh JL, Yao HY: Involvement of amygdala pathways in the influence of post-training intra-amygdala norepinephrine and peripheral epinephrine on memory storage. Brain Res. 1990, 508:225-233.

16. Hatfield T, McGaugh JL: Norepinephrine infused into the basolateral amygdala posttraining enhances retention in a spatial water maze task. Neurobiol. Learn. Mem. 1999, 71:232-239.

17. Ferry B, McGaugh JL: Clenbuterol administration into the basolateral amygdala posttraining enhances retention in an inhibitory avoidance task. Neurobiol. Learn. Mem. 1999, 72:8-12.

18. Huff NC, Wright-Hardesty KJ, Higgins EA, Matus-Amat P, Rudy JW: Context pre-exposure obscures amygdala modulation of contextual-fear conditioning. Learn Mem 2005, 12:456-460.

19. Roozendaal B, Castello NA, Vedana G, Barsegyan A, McGaugh JL: Noradrenergic activation of the basolateral amygdala modulates consolidation of object recognition memory. Neurobiol. Learn. Mem. 2008, 90:576-579.

*20. Barsegyan A, McGaugh JL, Roozendaal B: Noradrenergic activation of the basolateral amygdala modulates the consolidation of object-in-context recognition memory. Front. Behav. Neurosci. 2014, 8:160.

21. Yang F-C, Liang KC: Interactions of the dorsal hippocampus, medial prefrontal cortex 
and nucleus accumbens in formation of fear memory: difference in inhibitory avoidance learning and contextual fear conditioning. Neurobiol. Learn. Mem. 2014, 112:186-194.

22. Ardenghi P, Barros D, Izquierdo LA, Bevilaqua L, Schröder N, Quevedo J, Rodrigues C, Madruga $\mathrm{M}$, Medina JH, Izquierdo I: Late and prolonged post-training memory modulation in entorhinal and parietal cortex by drugs acting on the CAMP/protein kinase A signalling pathway. Behav Pharmacol 1997, 8:745-751.

23. Bevilaqua L, Ardenghi P, Schröder N, Bromberg E, Schmitz PK, Schaeffer E, Quevedo J, Bianchin M, Walz R, Medina JH, et al.: Drugs acting upon the cyclic adenosine monophosphate/protein kinase A signalling pathway modulate memory consolidation when given late after training into rat hippocampus but not amygdala. Behav Pharmacol 1997, 8:331-338.

24. Ferry B, Roozendaal B, McGaugh JL: Involvement of alpha1-adrenoceptors in the basolateral amygdala in modulation of memory storage. Eur. J. Pharmacol. 1999, 372:916.

25. Ferry B, Roozendaal B, McGaugh JL: Basolateral amygdala noradrenergic influences on memory storage are mediated by an interaction between beta- and alpha1adrenoceptors. J. Neurosci. 1999, 19:5119-5123.

26. Liang KC, Juler RG, McGaugh JL: Modulating effects of posttraining epinephrine on memory: involvement of the amygdala noradrenergic system. Brain Res. 1986, 368:125133.

*27. Quirarte GL, Roozendaal B, McGaugh JL: Glucocorticoid enhancement of memory storage involves noradrenergic activation in the basolateral amygdala. Proc. Natl. Acad. Sci. U.S.A. 1997, 94:14048-14053.

**28. Mclntyre CK, Hatfield T, McGaugh JL: Amygdala norepinephrine levels after training predict inhibitory avoidance retention performance in rats. Eur. J. Neurosci. 2002, 16:1223-1226.

This study shows that norepinephrine levels in the amygdala are elevated for 2 hours after an aversive inhibitory avoidance training experience and that norepinephrine responses of individual rats correlate with their later retention performance.

29. McGaugh JL: Memory consolidation and the amygdala: a systems perspective. Trends Neurosci 2002, 25:456-461.

*30. Lovitz ES, Thompson LT: Memory-enhancing intra-basolateral amygdala clenbuterol infusion reduces post-burst afterhyperpolarizations in hippocampal CA1 pyramidal neurons following inhibitory avoidance learning. Neurobiol. Learn. Mem. 2015, 119:3441. 
31. van Stegeren AH, Everaerd W, Cahill L, McGaugh JL, Gooren LJ: Memory for emotional events: differential effects of centrally versus peripherally acting beta-blocking agents. Psychopharmacology 1998, 138:305-310.

32. Abercrombie HC, Speck NS, Monticelli RM: Endogenous cortisol elevations are related to memory facilitation only in individuals who are emotionally aroused. Psychoneuroendocrinology 2006, 31:187-196.

33. Preuss D, Wolf OT: Post-learning psychosocial stress enhances consolidation of neutral stimuli. Neurobiol. Learn. Mem. 2009, 92:318-326.

**34. Cahill L, Gorski L, Le K: Enhanced human memory consolidation with post-learning stress: interaction with the degree of arousal at encoding. Learn Mem 2003, 10:270274.

This study shows that post-learning stress exposure in humans interacts with arousal level at encoding to selectively enhance memory of emotional, but not neutral, information.

35. Smeets T, Otgaar H, Candel I, Wolf OT: True or false? Memory is differentially affected by stress-induced cortisol elevations and sympathetic activity at consolidation and retrieval. Psychoneuroendocrinology 2008, 33:1378-1386.

36. Felmingham KL, Tran TP, Fong WC, Bryant RA: Sex differences in emotional memory consolidation: The effect of stress-induced salivary alpha-amylase and cortisol. Biol Psychol 2012, 89:539-544.

37. Zoladz PR, Peters DM, Cadle CE, Kalchik AE, Aufdenkampe RL, Dailey AM, Brown CM, Scharf AR, Earley MB, Knippen $C L$, et al.: Post-learning stress enhances long-term memory and differentially influences memory in females depending on menstrual stage. Acta Psychol (Amst) 2015, 160:127-133.

38. Nielson KA, Powless M: Positive and negative sources of emotional arousal enhance long-term word-list retention when induced as long as $30 \mathrm{~min}$ after learning. Neurobiol. Learn. Mem. 2007, 88:40-47.

39. Southwick SM, Davis M, Horner B, Cahill L, Morgan CA, Gold PE, Bremner JD, Charney DC: Relationship of enhanced norepinephrine activity during memory consolidation to enhanced long-term memory in humans. Am. J. Psych. 2002, 159:1420-1422.

40. Cahill L, Alkire MT: Epinephrine enhancement of human memory consolidation: interaction with arousal at encoding. Neurobiol. Learn. Mem. 2003, 79:194-198.

41. Bouret S, Sara SJ: Network reset: a simplified overarching theory of locus coeruleus noradrenaline function. Trends Neurosci 2005, 28:574-582.

*42. Sara SJ: The locus coeruleus and noradrenergic modulation of cognition. Nat. Rev. 
Neurosci. 2009, 10:211-223.

**43. Sara SJ, Bouret S: Orienting and reorienting: the locus coeruleus mediates cognition through arousal. Neuron 2012, 76:130-141.

A comprehensive review detailing the cognitive functions of noradrenergic signaling.

44. Nieuwenhuis S, Aston-Jones G, Cohen JD: Decision making, the P3, and the locus coeruleus--norepinephrine system. Psychol. Bull. 2005, 131:510-532.

45. de Martino B, Strange BA, Dolan RJ: Noradrenergic neuromodulation of human attention for emotional and neutral stimuli. Psychopharmacology 2008, 197:127-136.

*46. Hermans EJ, van Marle HJF, Ossewaarde L, Henckens MJAG, Qin S, van Kesteren MTR, Schoots VC, Cousijn H, Rijpkema M, Oostenveld R, et al.: Stress-related noradrenergic activity prompts large-scale neural network reconfiguration. Science 2011, 334:11511153.

**47. Seeley WW, Menon V, Schatzberg AF, Keller J, Glover GH, Kenna H, Reiss AL, Greicius MD: Dissociable intrinsic connectivity networks for salience processing and executive control. J. Neurosci. 2007, 27:2349-2356.

Seminal paper that introduced the concept of a "salience network", a large-scale intrinsic connectivity network delineated using resting-functional MRI.

48. Menon V: Large-scale brain networks and psychopathology: a unifying triple network model. Trends Cogn. Sci. 2011, 15:483-506.

49. Hamann SB, Ely TD, Grafton ST, Kilts CD: Amygdala activity related to enhanced memory for pleasant and aversive stimuli. Nat. Neurosci. 1999, 2:289-293.

50. Cahill L, Haier RJ, White NS, Fallon J, Kilpatrick L, Lawrence C, Potkin SG, Alkire MT: Sexrelated difference in amygdala activity during emotionally influenced memory storage. Neurobiol. Learn. Mem. 2001, 75:1-9.

51. Canli T, Zhao Z, Brewer J, Gabrieli JD, Cahill L: Event-related activation in the human amygdala associates with later memory for individual emotional experience. $J$. Neurosci. 2000, 20:RC99.

52. Cahill L, Uncapher M, Kilpatrick L, Alkire MT, Turner J: Sex-related hemispheric lateralization of amygdala function in emotionally influenced memory: an FMRI investigation. Learn Mem 2004, 11:261-266.

53. Kensinger EA, Corkin S: Two routes to emotional memory: distinct neural processes for valence and arousal. Proc. Natl. Acad. Sci. U.S.A. 2004, 101:3310-3315. 
54. Fastenrath M, Coynel D, Spalek K, Spalek K, Milnik A, Gschwind L, Roozendaal B, Roozendaal B, Papassotiropoulos A, Papassotiropoulos A, et al.: Dynamic modulation of amygdala-hippocampal connectivity by emotional arousal. J. Neurosci. 2014, 34:1393513947.

**55. Dolcos F, Labar KS, Cabeza R: Interaction between the amygdala and the medial temporal lobe memory system predicts better memory for emotional events. Neuron 2004, 42:855-863.

This study shows in humans how amygdala and hippocampus interact during emotional memory formation.

56. Richardson MP, Strange BA, Dolan RJ: Encoding of emotional memories depends on amygdala and hippocampus and their interactions. Nat. Neurosci. 2004, 7:278-285.

57. Strange BA, Dolan RJ: Beta-adrenergic modulation of emotional memory-evoked human amygdala and hippocampal responses. Proc. Natl. Acad. Sci. U.S.A. 2004, 101:1145411458.

58. van Stegeren AH, Goekoop R, Everaerd W, Scheltens P, Barkhof F, Kuijer JPA, Rombouts SARB: Noradrenaline mediates amygdala activation in men and women during encoding of emotional material. Neurolmage 2005, 24:898-909.

59. Rimmele U, Lackovic SF, Tobe RH, Leventhal BL, Phelps EA: Beta-adrenergic Blockade at Memory Encoding, but Not Retrieval, Decreases the Subjective Sense of Recollection. J. Cogn. Neurosci. 2016, 28:895-907.

60. Onur OA, Walter H, Schlaepfer TE, Rehme AK, Schmidt C, Keysers C, Maier W, Hurlemann R: Noradrenergic enhancement of amygdala responses to fear. 2009, 4:119-126.

61. Rasch B, Spalek K, Buholzer S, Luechinger R, Boesiger P, Papassotiropoulos A, de Quervain DJ-F: A genetic variation of the noradrenergic system is related to differential amygdala activation during encoding of emotional memories. Proc. Natl. Acad. Sci. U.S.A. 2009, 106:19191-19196.

62. Cousijn H, Rijpkema M, Qin S, van Marle HJF, Franke B, Hermans EJ, van Wingen G, Fernández $\mathrm{G}$ : Acute stress modulates genotype effects on amygdala processing in humans. Proc. Natl. Acad. Sci. U.S.A. 2010, 107:9867-9872.

63. de Quervain DJ-F, Kolassa I-T, ErtI V, Onyut PL, Neuner F, Elbert T, Papassotiropoulos A: A deletion variant of the alpha2b-adrenoceptor is related to emotional memory in Europeans and Africans. Nat. Neurosci. 2007, 10:1137-1139.

*64. Seidenbecher T, Laxmi TR, Stork O, Pape H-C: Amygdalar and hippocampal theta rhythm synchronization during fear memory retrieval. Science 2003, 301:846-850. 
65. Ghosh S, Laxmi TR, Chattarji S: Functional connectivity from the amygdala to the hippocampus grows stronger after stress. J. Neurosci. 2013, 33:7234-7244.

66. Süer C, Ozesmi C, Temoçin S, Doğan P, Ciliv G: The effects of immobilization stress on electrodermal activity and brain catecholamine levels in rats. Int. J. Neurosci. 1992, 65:91-101.

**67. Popa D, Duvarci S, Popescu AT, Léna C, Paré D: Coherent amygdalocortical theta promotes fear memory consolidation during paradoxical sleep. Proc. Natl. Acad. Sci. U.S.A. 2010, 107:6516-6519.

This study demonstrates that theta coherence between amygdala and hippocampus during sleep predicts long-term expression of fear memories.

68. Carr MF, Jadhav SP, Frank LM: Hippocampal replay in the awake state: a potential substrate for memory consolidation and retrieval. Nat. Neurosci. 2011, 14:147-153.

69. Karlsson MP, Frank LM: Awake replay of remote experiences in the hippocampus. Nat. Neurosci. 2009, 12:913-918.

70. Tambini A, Ketz N, Davachi L: Enhanced brain correlations during rest are related to memory for recent experiences. Neuron 2010, 65:280-290.

71. van Kesteren MTR, Fernández G, Norris DG, Hermans EJ: Persistent schema-dependent hippocampal-neocortical connectivity during memory encoding and postencoding rest in humans. Proc. Natl. Acad. Sci. U.S.A. 2010, 107:7550-7555.

72. Tambini A, Davachi L: Persistence of hippocampal multivoxel patterns into postencoding rest is related to memory. Proc. Natl. Acad. Sci. U.S.A. 2013, 110:19591-19596.

73. Staresina BP, Alink A, Kriegeskorte N, Henson RN: Awake reactivation predicts memory in humans. Proc. Natl. Acad. Sci. U.S.A. 2013, 110:21159-21164.

**74. Hermans EJ, Kanen JW, Tambini A, Fernández G, Davachi L, Phelps EA: Persistence of amygdala-hippocampal connectivity and multi-voxel correlation structures during awake rest after fear learning predicts long-term expression of fear. Cereb. Cortex 2016, doi:10.1093/cercor/bhw145.

This paper shows that the persistence of learning-related patterns of amygdala activity during post-learning rest predicts strength of consolidation in humans.

75. de Voogd LD, Fernández G, Hermans EJ: Awake reactivation of emotional memory traces through hippocampal-neocortical interactions. Neurolmage 2016, 134:563-572.

**76. Ritchey M, McCullough AM, Ranganath C, Yonelinas AP: Stress as a mnemonic filter: Interactions between medial temporal lobe encoding processes and post-encoding 
stress. Hippocampus 2017, 27:77-88.

This paper shows that stronger cortisol reactivity to post-learning stress is associated with stronger subsequent memory effects of amygdala and hippocampus activity during encoding, thus providing the first demonstration of an encoding-consolidation interaction in humans.

77. Wilhelm I, Wagner U, Born J: Opposite effects of cortisol on consolidation of temporal sequence memory during waking and sleep. J. Cogn. Neurosci. 2011, 23:3703-3712.

78. van Marle HJF, Hermans EJ, Qin S, Overeem S, Fernández G: The effect of exogenous cortisol during sleep on the behavioral and neural correlates of emotional memory consolidation in humans. Psychoneuroendocrinology 2013, 38:1639-1649.

**79. Strange BA, Hurlemann R, Dolan RJ: An emotion-induced retrograde amnesia in humans is amygdala- and beta-adrenergic-dependent. Proc. Natl. Acad. Sci. U.S.A. 2003, 100:13626-13631.

This paper shows a retrograde amnesia for neutral items preceding emotional oddballs. It further shows that this effect is abolished after beta-adrenergic receptor blockade, and is absent in patients with amygdala lesions.

80. Sakaki M, Fryer K, Mather M: Emotion strengthens high-priority memory traces but weakens low-priority memory traces. Psych. Sci. 2014, 25:387-395.

**81. Mather M: Norepinephrine ignites local hot spots of neuronal excitation: How arousal amplifies selectivity in perception and memory. 2015, [no volume].

Mather et al. propose a mechanism that can explain both enhancing and impairing effects of norepinephrine on cognition.

82. Terburg D, Morgan BE, Montoya ER, Hooge IT, Thornton HB, Hariri AR, Panksepp J, Stein DJ, van Honk J: Hypervigilance for fear after basolateral amygdala damage in humans. Transl Psychiatry 2012, 2:e115-e115.

83. Cahill L, Babinsky R, Markowitsch HJ, McGaugh JL: The amygdala and emotional memory. Nature 1995, 377:295-296.

84. Bechara A, Tranel D, Damasio H, Adolphs R, Rockland C, Damasio AR: Double dissociation of conditioning and declarative knowledge relative to the amygdala and hippocampus in humans. Science 1995, 269:1115-1118.

85. Klumpers F, Morgan B, Terburg D, Stein DJ, van Honk J: Impaired acquisition of classically conditioned fear-potentiated startle reflexes in humans with focal bilateral basolateral amygdala damage. 2015, 10:1161-1168. 
86. Hermans EJ, Battaglia FP, Atsak P, de Voogd LD, Fernández G, Roozendaal B: How the amygdala affects emotional memory by altering brain network properties. Neurobiol. Learn. Mem. 2014, 112:2-16. 


\section{Figure Legends}

\section{Figure 1}

Norepinephrine levels in the amygdala are elevated for 2 hours after footshock stimulation during inhibitory avoidance training and correlate with 24-hour retention performance. Percent of baseline norepinephrine following inhibitory avoidance training is graphed for each individual rat. The key notes retention latencies 24 hours after the training session (maximum latency $=600$ seconds). Longer retention latencies are interpreted as indicating better memory. Correlation values for the first five post-training samples varied from +0.75 to +0.92 . Adapted from McIntyre et al. [28].

\section{Figure 2}

Interaction between phasic noradrenergic signaling during encoding and sustained noradrenergic activity during memory consolidation. This figure illustrates the core proposal of this paper that sustained noradrenergic activity during consolidation enhances memory for events that were associated with phasic noradrenergic signaling during encoding, but may impair memory for events that were not preceded by phasic noradrenergic signaling during encoding. Both the phasic and sustained noradrenergic effects depend on the amygdala. 


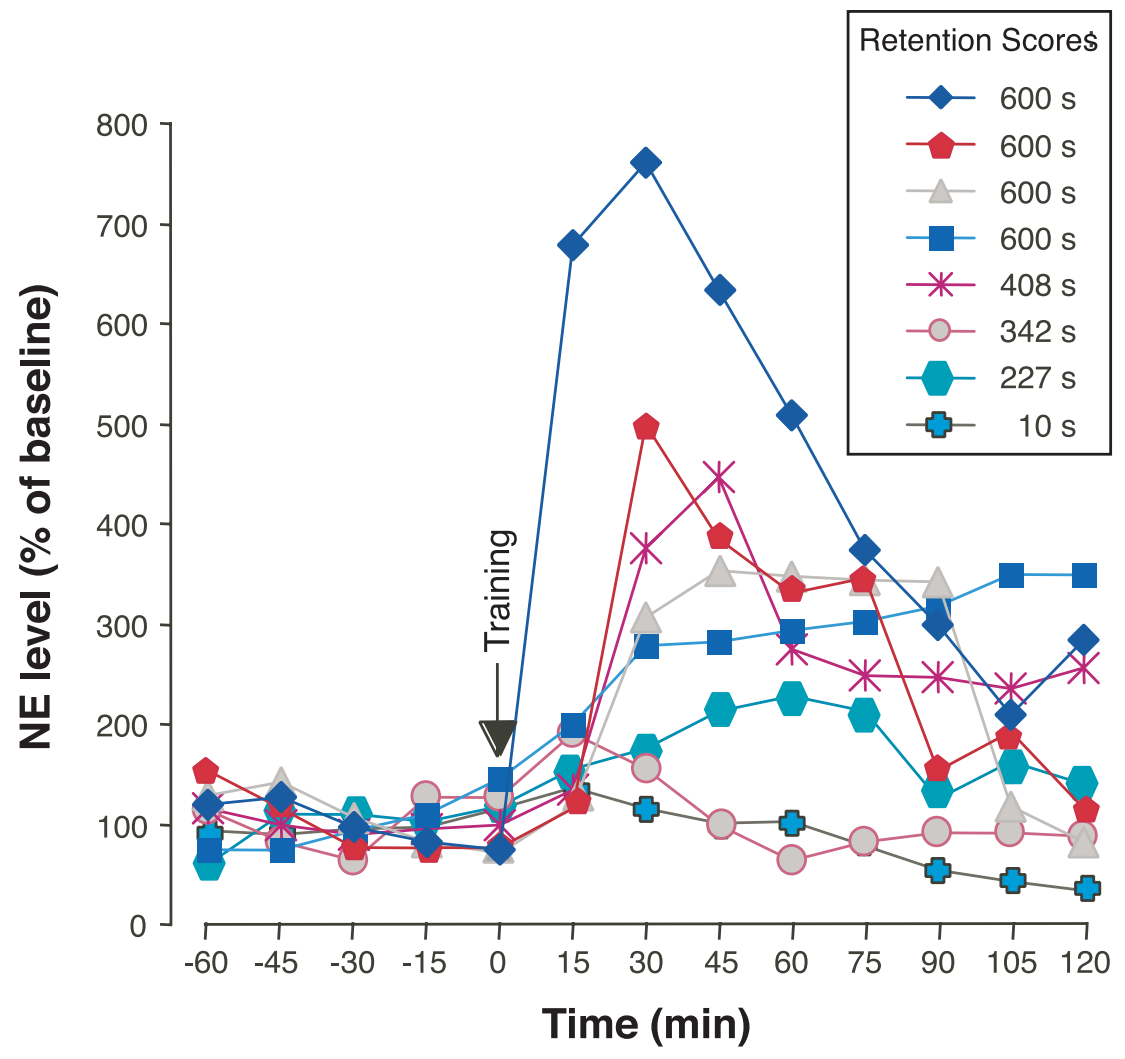




\section{Phasic NE \\ Amygdala}

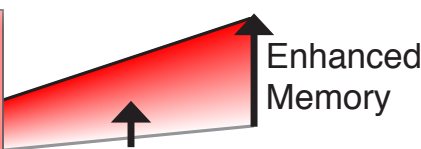

Post-learning Stress \&

No phasic Amygdala

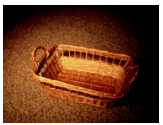
Sustained NE NE 